\title{
On the design of a dynamic reconfigurable network switch
}

\author{
Gerard J.M. Smit, Pat J.M. Havinga. Pierre G. Jansen \\ University of Tivente, Dept. of Computer Science \\ P.O. Box 217, 7500 AE Enschede, the Netherlands \\ c-mail: smit@cs.titwente.ni
}

Keywords: Kautz graphs, programmable architecture, network switch, Field Programmable Gate Array (FPGA).

\section{Introduction}

In this paper we will presemt a reconfigurable nerwork switch for multi-computer syslems. $\Lambda$ multi-computer system is defined as a collection of linked node computers (abbreviated as nodes), in which the nodes communicate via message passing [Dally 87]. As a communication network for our system we use a Keutz network [Kautz 68]. Each node consists of three autonomous sub-systems: a Computation Processor (CP) with lacal memory a Router (R) and a Network Switch (NS). The Computation Processor is a standard off-the-shelf type of processor that executes application programs. The Router provides the interface to other nudes and implements the communication protocol. The roules in the network are generated by the Roule Gencrator (RO) [Smit O]b] which is part of the Routcr. The Network Switch und Router are implemented with Field Programmable Gate Array (FPGA) technology [Xilinx 91]. In this technolugy the gate arrays can be re-programmed an unlimited number of times. Essential in our approach is that FPGAs are used as dynamic programmable units, which function can be changed on-the-fly under program control. Therefore they can be used in designs where hardware is changed dynamically, or when hardwate must be adapted to different user applications.

In order to provide fuli connectivity in a network of computers, routing mechanisms must be used. These mechanisms must satisfy a number of requirements such as: iree of deadlocks, no starvation, low latency and high throughput. Wellknown routing mechanisms are store-and-forward, wormhole routing, virtual cut-through. When a message in the network is unable to proceed because some resource it needs is held by other mexsiges (collision) some action has to he taken. Possible options are: blocking the messigge, buffering the message prior to the noxk where the cotilision ocesurs, dropping and retransmission of the messige, or misrouting the message to a free link. It is known that some of these solutions bavt a potential danger of deadlock. There are a number of mechanisms for avoiding conmunication deadlocks ila networks. Most teclınjutets such as virtual networks and clatss climbing. are trised on breaking loups in the dependency graph [Dally 87 ]. Dropping and retransmission of messages is inherently free of deadlocks. This last method, also called the method of the nosy worms [Whobrey 88 , is used in the configuration presented in this paper. The method of misrouting as used in the ConnectionMachine is not applicable, because misrouting is not deadlock free and in case of a misroute a new route has to be computed.

The main function of the Network Switch is to foute messages in the communication network. The NS does not com:pute nor change the contents of the messages. It only uses the information in the route field of a message to control the destination of that message. The route itself is computed by the Router and the Routc Generator. The NS communicates with other switches and the Router via links. The NS manages incoming messages autonomously: establishes the route and passes the data of the messages through or returns a message if all links are busy. Because the switches are implemented in FPGA technology, the precise message format is not fixed by design. For instance the decision for fixed or variable tength messages can be taken at a later stage in the design process.

The Router assembles outgoing messages, sends these messages to the NS and handles incoming messages. The data of the messages generally comes from and goes to the local memory. The Router interacts with the memory without intervention from the CP. The Route Generator, a sub-unit of the Router, is a logic unit that generates the of node disjoint routes of a Kautz graph, given a source and a destination node (see section 2). If the NS of the source reports that a message did not reach the destination (due to congestion or link/node failures), the Router reads a new route from the Route Generator and assembles a new message. If all node disjoint paths have been tried the $C P$ is informed. The $C P$ can decitle to try again later after a random delay [Whobrey 88].

\section{Kautz networks}

We use Kautz networks in our propect because these networks have interesting properties [Bermond \$9]. Particubarly. they interconnect considerahly more nodes than the usual topologies, and they have a small diameter, and a snall and fixed degrec. Furthermore they are highly fiult tolerim, admit selfrouting and ci:n cmbed standird computation 


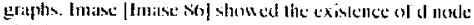
disjoint paths between any pair of nodes in a Kautz graph of $\mathrm{N}=\mathrm{d}^{\mathrm{k}}+\mathrm{d}^{\mathrm{k}-\mathrm{k}}$ nodes. These proportics makes Katz graphs suitable as an interconnection network for large scale parallel computer systcms.

Definition of Kautz graph.s [Kautz 68].

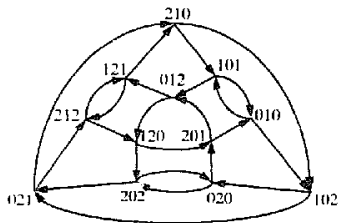

Figs. 1: Example of a Kaut? graph $(K(2,3))$.

The Kauz digraph K(d.k) with in-degree and out-degree d and diameter $k$ is the digraph whose vertices are labelled with wards $\left(x_{1} \ldots . . x_{k}\right)$ of length $k$ from an alphabet of $u+1$ leters by temoving thowe words in which there are two consteulive identical letters $\left(x_{j} \neq x_{j+1}\right.$. lin $\left.1 \leq \mathrm{i} \leq k-1\right)$. There is an are from a vertex $x$ lo a vertex $y$ if and only it the last $k-1$ letters of $x$ ate the sime at the lisst $k-1$ ?allers of $y$. A straightiorward generic route of length $\mathrm{k}$ can be found hy simple concatenation of solurce and destination word. However thice may ho roules wilh length $<k$ [Smit 9] a].

Fxample i (sec tig. 1)

In the graph we find the soutc $\left.R_{2}=<12030\right)>$ from

(120) to (201) via node $(2(12)$ and (0)(1). This reutte has length $3(=k)$.

The shortest route is $R s=\langle 1$ Col $1>$ of kenglt 1 .

Tahle 1 comparex Kautz digraphs w ith de Bruijo" diyraphs f de Brujign 46] and the binary hypetcube [llillis 85]. [Stite 85]. The de Brtijn digraph has been selected because its definition is closely related tu Katuly digraphs.

The difierence between a de Bruijn digraph $B(d . k)$ and a Kauty digraph $\mathbf{K}(d, k)$ in that in a de Bruign digraph two con secutive leters in the word representing a particular vertex mat be equal. As a somsequenct. this digraph contains selt losps.

$\begin{array}{lcccc}\quad d=k=4 & d=k=6 & d=k=8 & \text { number ol nodes } \\ \text { hypercube } & 16 & 6.4 & 250 & N=2^{h} \\ \text { de Bruijn } & 16 & 729 & 655.36 & N=d^{k} \\ \text { Katu } & 24 & 972 & 51920 & N=d^{k}+d^{k-1}\end{array}$

Tahle l.: Number af aoves of some graphs.

Sinte that for the de Bruifil and Kauly digraphs the out-degree and in-degroc are hill the degree mentioned in the ta-

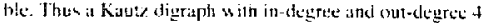

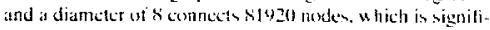

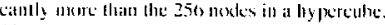

The Router cienerator as described in [Smit Y/b] generates the d node disjoint routes with increasing length. The routes are as short as possible and liee of loops.

\section{The Network Switch}

A designer of communichtion systems for multi-computers is faced with conllicting demands, due to varying application requirements, fast changing technology, etc. Experiences with existing parallel machines and simulations have shown for instance that not onc single routing mechanism is optimal tor all kinds of applications. In the case of low or medium communicaton tralfic, circuit switching or wormhole routing seem to have advantages over store-and-forwarding. But in case of in'tensive communication as for instance due to frequent broadcasting, the store-and-forward mechanism is more adequate [Seidel 89 ). If a communicalion network is kesigned with full-custom VLSI components, the designer is forced to make crucial decisions early in the design process.

In out design the Network Switch and Router are implemented with Fijeld Frogrimmahle Gate Army (IPG $\wedge$ ) tech. nology. This kechnology allows the gate arrays to he reprogrammed for an unlimiled number of times. Therefore they ite suited lor designs in which the lunetions of the hardware: necals adaplations in order to meel changing application requirements. This has a number of advantages, sach as:

- The selection of the most suighle communication mechanism can be posponed 16 a later stige of the design.

- The system designer or applicition programmer can 'esign applicalion specific eommunication primitives and mechanisms. Knowledge of the commonication strictule of the computation can be used to tune the netWork to the recyuirements of the eompulation on the-fly. Due in its thexibility the system ein be used in a wide varjety of applicatisms, ranging from high speed ommpulitions to dedicited reat-time applications.

- The design cyele of IPCAs is very short. Minor dexign shanges can be mode instantaneously.

- The cosit ol a protetype is very lew. FPGAs are standard compuments, nu design costs lor full-custom components.

In this paragraph ne prescon a possible Nelwork Switch coitfiguratiom. Tilc mont important design decisions are:

Eni-directional links of I 2 bits widt.

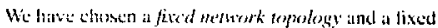

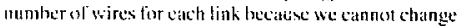
the phesical wiring of the sysfem dynamically, We dis' nom-fole retaling [Dally s7j. This type of routing suits mell to rouling in hintz networks and gives a bow hateney. An soon is is Vetwork Su itch reakls the header of a message, containitg the soute inlirmation, it 
seleces the next liuh ou the resule and fiswards lhe remaining part of the messiage dow th that link. Each NS consumes ane byte of the route.

- To avoid deadlock we use the nosy worms protocol [Whobrey 88]. If a messiage is blocked it is recoiled to the source. Becauses a Kautz network has d node-disjoint routes we expect that the probability of a collision is acceptable.

- As the amount of memory in a FPGA is relatively small the store-and-forward routing mechan ism seems less obvious. Although the local memory could be used as a butferspace for store-and-forward routing. Store-andforwast has a latency that is proportional to the product of packet length and number of hops. So worm-hole routing or virtual cut-through are more appropriate for our system.

- All Network Switches synct.ronize with each other via two synchronjzation signals per link.

A message consists of: a route field, a variable length data field and an End of Data marker. The route fieid of the packet defines precistly the route the meswige takes from source to destination.

As soon ats a NS reads the hender of a message, containing the route information, it selects the next link on the route, "consumes" the used route information and forwards the remaining part of the mestage down that link. lowever. if the output lin' is occupied it returns a negative acknowledge (NACK). Each NS between souree and destinalion consumes one byte of the roble until the message arrives at the NS of the destination. Now the route ficld of the message is emply and the mesesge is delivered at the Router of the dexlination. The end of the data is indicated with an Enal Of Data (EOIJ) mark.

We assume that the Router of the destination never refuses it incoming message forever.

If the Router of the source receives a NACK (due lu congestion or link/node failures). the Router assembles a new messhat: with another node disjoint route. If all node disjoint piths hive heen tried, the C.P is nutified.

\section{Implementation}

Fig. 2 shows the internal structure of the datidpath of the Network Switch. The switch has 3 input and 3 output links. One of the input links and one of the output links is connested to the Rouler. With this switch several physical networks with in-degrec and wut-clegree 2 can be buill, such as:

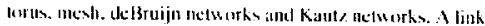

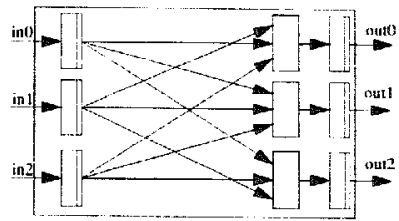

Fig. 2: Internal structuie of the Network Switch.

consists of the following 12 uni-directional signals: 8 data bits, I ype bit, a NACK signal and 2 synchomization signals $\left(\mathrm{cl}_{\mathbf{j}}\right.$ and $\mathrm{cla}_{\mathbf{i}}$ ). The type bit is used to indicate the start of a message and the end of the message (EOD). The NACK signal goes in the direction opposite to the data.

In the oresented system we use an externally asynchronous and internaly clocked design methodology.

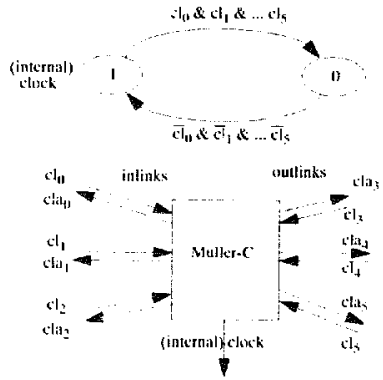

Fig. 3: State ditigram and block diagram of a Muller-i" element.

The design process of synchronous sircuits is much wasis compared to the design of asy nchrombtas eircuits. Moresover. the structure of FPCA As suits well to synchronous designs. However in large scale multi-emputers there is a difficult and otien underesimaled problem of elex's distribulion. In our dexign we found a compromise that invokus the advit;:tayes of hoth the symhromous atd anvichromus desizn methodologies. Irut without many of their disidvantages. Frem the perspetive af the external worlal such a syst:

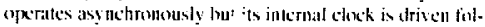
luwing a specific handshate protecol flinder $\$ 1$. in this

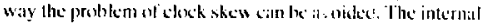
ches is gencrated iny a Muller-C clement, alsos sille: - Ren-

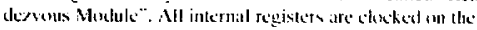




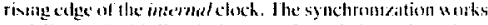
as follows: the Muller-C element of a switch receives the clock signals ( $\mathrm{ct}_{\text {i }}$ ) of all neighbor switches (see fig. 3 ). When all clock signals are asserted it negates the internal clock.

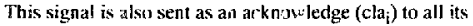
neighbors. If a $\mathrm{C}$ element has received all the acknowledges (all cligna sig negatcd) it asserts the internal clock and so the cla signals again. To assure the enrtect synchronization of the switches all switches must respond to the asisertion and negation of the $\mathrm{cl}_{\mathrm{i}}$ signals of the neightors even if tiby hive nothing to send.

\section{Realization}

The above mentioned network switch configuration has heen realized with a XC3042 Field Proytammable Gate Array of Xilinx [Xilinx 91]. It uses a decentralized control. so six bytes can be handled simultaneously by a switch in one "clock cucle". Table 2 gives some results of the realization. The clock speed jis derived from the outpat of the hardware simulator of Xilinx. The implementation was lirst described in VHDL and then simulated. After that the switch was automalically synthesized by the VHDL.synthesizer irom Viewlogic [Viewlogic 90]. Due to the high level specification in VHDL and the powerful syathesizer, the whers dexign process took only 3 wecks. We expect that the speed uf lite switein can be improved significantly by a careful (manual) redenign.

Number of $\left(1.3^{1)}\right.$ s usud: 126

Number of 10 pins used: 7.5

ivailable: 144

ivailable: 96

Number of logic levels used: 4

Maximum "cluck" speed: 10 Mhy.

Transter rale per link: so Misitsec.

lable 2: Resulis of a realization with a X(:3042 FP(AN.

\section{Conchusism}

In this paper we have presented the design of a Network Switch for a multi-computis. It can efficiently support different styles of communicution. such as worm-hole resting store-and-lorward routing and virtual cul-through. Each node of the multi-compiner consists of three autonomous subsystems: the Computation Processor, the Router, and the Network Switch. The Router and the Network Switch art implemented with FPG a technology: This implies that the system designer can alter the communication mechanism. even in between the execution of two application programs. The communication network is hased an a Katuz topolingy. Katue graphs form a clans of interconnection networks with interesting properties such as: small diameter. large number of nodes $\left(\mathrm{N}=\mathrm{d}^{\mathrm{h}}+\mathrm{d}^{\mathrm{k}-\mathrm{l}}\right)$. the degrete is indepenutent of the network size. the network is lault-telerant. it wan emhed standard compotation graphs and has a simple routing algorithin.

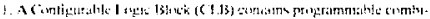

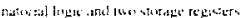

The presented network switch uses worm-hole routing, and a simple deadlock avoidance protocol. Worm-hole routing suits well to routing in Kautz networks and gives a low latency. All Network Switches synchronize with each other. such that a global clock is not requited.

Processors communicate with non neighboring nodes directly without involving the Computation Processor and Routers at the intervening nodes.

\section{Refernees}

[Bermond 89] Bermond J.C., Homobono N., Peyrat C.: "I arge Fault-Tolerant Interconnection Netwarks", Graphs and Combinatorics, 1989.

[Dally 87] Dally W.J.: "A VLSI Architecture for Concurrent Data Structures", Ph.D. thesis, Computer Science, California Institute of Technology, 1987.

[de Bruijn 46] de Bruiju N.G.: "A combinatorial prablem"; Koninklijke Nederlandse Academic van Wetenschappen Froc. A49, pp 758-764; 1946.

[Hillis 85] Hillis W.D.: "ine connection machine"; The MIT press: 1985.

[Imase 8í] Imase M., Soneoka T., C.kata K.: “A faulttolerant processor intercinnection network" (original in Japanest), translated in Systims and Computers in Japan, vol 17. no 8 pp 21-30, 1986.

[Kautz 68] Kautz W.H.: "Bounds on direcled (d.k) graphs. Theory of cellular logic networks anit machines", A FCRL-68-1668 Final report, pp 20-20, 1968.

[Seiz 85] Scitz C.L.:. "The cosmic cube"; Comm, ACMl, Vol 28, no 1, jan. 1985.

[Smit 91a] Smil G.J.M.. Havinga P.J.M., Jansen P.G.: "An algorithm for generating; node disjoint routes in Kautz digraphs". Proccediags Fifth International Paralle! Processing Synoposism. pp. 102-167. viay 1991.

[Smil 9lb] Smit G.J.M. Wuvinga P.I.M., Jansen P.G., de Buer F.. Mulenkump F..: "On hardware for generatio:s routes in Kautz graphs". procesedings Euromicro91, 1991 .

['Tinder 91] Tinder R.F.: -Digital Fngineering Desigh, A Mutern Approach". pp. 638.646. Prentice Hall, 1991.

[VHDL 87 ] "VIHDL Language Relerence Manual", IEEESID- IOT6-1957, IHF1: Computer Suctely.

[VIFW Wheic 90] "*VHDI,-Designer User's Givide", VIEW/ngie Systems. Ins. April 1990).

[Whobrey $\mathrm{K} \alpha$ ] Whobrey D.: "A communicitions chip for multiprocessurs". Proce. CONPAR is np 464-473. 19หัร.

[Xilinx 41] "The Programmible Ciat Aray Data Book", Xilinx Inci. 1991 . 